\title{
APLICACIÓN DE ESTRATEGIAS HEURÍSTICAS EN LA SOLUCIÓN DE PROBLEMAS QUE SE MODELAN MEDIANTE ECUACIONES ALGEBRAICAS EN ESTUDIANTES DE UNA INSTITUCIÓN EDUCATIVA
}

\section{Application of Heuristic Strategies in the Solution of Problems Modeled by Means of Algebraic Equations in Students of an Educational Institution}

\section{Andrea Peña-Sureda ${ }^{1}$}

andreapena@pucpr.edu

\section{Abner Colón-Ortiz ${ }^{1}$}

abner_colon@pucpr.edu

Iris Ramos-Rullán'

iramosrullan@pucpr.edu

\section{Resumen}

El propósito de la investigación fue proveer estrategias aplicables en el proceso de solución de problemas que se modelan mediante ecuaciones algebraicas para determinar si hubo diferencia significativa en el rendimiento académico de los grupos bajo investigación. Se realizó un estudio piloto y un análisis de dificultad y discriminación. La muestra de los tres grupos fueron 26 estudiantes matriculados en un curso general de matemáticas como requisito de su programa de estudios. Se le administró una preprueba y una posprueba al grupo de control 1 (C1) y al grupo experimental (E). La posprueba se administró a los grupos C1, C2 y E. Se llevó a cabo el tratamiento en el que los grupos de control recibieron enseńanza tradicional y el grupo experimental, enseñanza basada en la aplicación de estrategias heurísticas. Se realizó una prueba de normalidad y un análisis de discrepancia y dificultad. Se calculó la media de la administración de la preprueba. Se concluyó que C1 y E tenían similar rendimiento y conocimiento en la solución de problemas y ecuaciones algebraicas al inicio de la investigación, y que existió una diferencia significativa después del tratamiento en los grupos $\mathrm{C} 1 \mathrm{y}$ $\mathrm{E}$, se mostró que los grupos $\mathrm{C} 1$ y $\mathrm{E}$ eran homogéneos, y que existió una diferencia significativa entre los tres grupos participantes en la posprueba de rendimiento académico. Se aportó un instrumento de investigación, un módulo educativo para estudiantes y una guía estratégica para el profesor.

Palabras clave: ecuaciones, heurística, solución de problemas.

\section{Abstract}

The purpose of the research was to provide strategies applicable in the process of troubleshooting algebraic equations to determine whether there was a significant difference in the academic performance of the groups under investigation. A pilot study and a problem and discrimination analysis were conducted. The sample of the three groups was 26 students enrolled in a general math course as a requirement of their curriculum. A pre- and post-test was given to the control group $1(\mathrm{C} 1)$ and the experimental group (E). The post-test was administered to groups $\mathrm{C} 1, \mathrm{C} 2$ and E. Treatment was carried out where the control groups received traditional teaching and the experimental group teaching based on the application of heuristic strategies. A normality test and a discrepancy and difficulty analysis were performed. The average pretest administration was calculated. It was concluded that $\mathrm{C} 1$ and E had similar performance and knowledge in problem solving and algebraic equations at the beginning of research, there was significant difference after treatment in groups $\mathrm{C} 1$ and $\mathrm{E}$, it was shown that groups $\mathrm{C} 1$ and $\mathrm{E}$ were homogeneous, and that there was significant difference between the three groups participating in the posttest of academic performance. A research tool, an educational module for students and a strategic guide for the teacher were provided.

Keywords: equations, heuristics, problem solving.

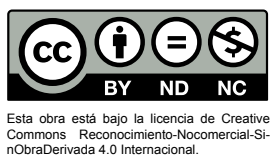

ISSN (impreso): 2636-2139 


\section{Introducción}

En el año 1980, el Consejo Nacional de Maestros de Matemáticas (NCTM, por sus siglas en inglés), en la publicación An Agenda for Action, recomendó que la solución de problemas fuese el eje central de la enseñanza y aprendizaje de las matemáticas en las escuelas. El desarrollo de la habilidad de cada estudiante para la solución de problemas es esencial para su productividad como ciudadano (NCTM, 1989). Los problemas tienen diferentes características y normalmente su solución requiere diferentes destrezas y estrategias. Los estudiantes deben aprender matemáticas y solucionar problemas construyendo nuevos conocimientos sobre los fundamentos previamente adquiridos (NCTM, 2019). Muchas de las dificultades que surgen en el proceso de solución de problemas se deben a las diferentes características que tienen y cómo su estructura, naturaleza, contexto y representación influyen en el método de solución (Oladunni, 1998). Santaló (1995) señaló que el enseñar matemáticas es equivalente a enseñar a solucionar problemas: cuando se estudia esta materia, se solucionan problemas. El propósito de enseñar la solución de problemas es que los estudiantes puedan desarrollar una habilidad general de solucionar problemas de la vida real y aplicar la matemática aprendida a situaciones de la vida real (Guzman Gurat, 2018).

Lesh y Zawojewski (2007) establecieron que la solución de problemas es como un proceso de interpretar una situación matemática al incorporar la formación y revisión para comprender conceptos básicos de matemáticas. Si se admite que solucionar un problema genuino no es tan solo seguir unos pasos algorítmicos, entonces es necesario aceptar el uso de estrategias generales para la solución de problemas y determinar cómo se utilizan las mismas (Novotná et al., 2014). De acuerdo con Espinoza (2017), la solución de problemas se ha convertido en una parte esencial de los procesos de enseñanza y aprendizaje de matemáticas. Esta investigación tuvo como propósito proveer estrategias aplicables en el proceso de solución de problemas que se modelan mediante ecuaciones algebraicas.
La presente investigación se realizó por medio de un enfoque cuantitativo con un estudio cuasiexperimental. En la misma se aplicaron estrategias heurísticas y enseñanza tradicional para la solución de problemas que se modelan mediante ecuaciones algebraicas. El grupo experimental recibió enseñanza no tradicional con la aplicación de estrategias heurísticas y los grupos de control recibieron enseńanza tradicional para la solución de problemas que se modelan mediante ecuaciones algebraicas. Por último, se analizó la diferencia entre los grupos de control sometidos a enseñanza tradicional y el grupo experimental sometido a la aplicación de estrategias heurísticas para la solución de problemas que se modelan mediante ecuaciones algebraicas.

\section{Revisión de la literatura}

El objetivo de la heurística es estudiar métodos y reglas para el descubrimiento y la invención (Polya, 1945). De acuerdo con Calucag (2016), un método heurístico está relacionado con un proceso de descubrimiento controlado en el que el aprendizaje se lleva a cabo cuando el estudiante reacciona y actúa desarrollando un plan en el que se corroboren los supuestos. Romberg (1998) señaló que el enfoque de la enseñanza debe requerir que el estudiante investigue, formule, razone, lea y utilice estrategias para resolver problemas, demuestre aseveraciones y reflexione sobre cómo se utilizan las matemáticas. Tradicionalmente, en la escuela se ha identificado la enseñanza y el contexto en el que se desarrolla la enseñanza como dos cosas completamente distintas (Pehkonen et al., 2013). La enseñanza tradicional está centrada en el aprendizaje de hechos; sin embargo, utilizar nuevos métodos es necesario para aprender nuevos conocimientos (Pehkonen et al., 2013). Según Stinson y Claus (2000), la enseñanza tradicional está definida como largas clases en las que los maestros aportan información por un tiempo determinado, con una estructura física que se compone de un salón con sillas ordenadas en forma de filas, una pizarra al frente de las sillas y clases magistrales en el salón de clases; presentaron la enseñanza tradicional como un proceso de aprendizaje aburrido para los estudiantes del siglo XXI. 
Por otra parte, Pereira-Mendoza (1980) llevó a cabo una investigación sobre el efecto de la enseñanza de heurística en la habilidad de estudiantes de décimo grado para solucionar problemas matemáticos. Según este investigador, un grupo de niños se autoinstruyó durante 10 días con cuadernillos en cómo aplicar métodos heurísticos para examinar casos y para la solución de problemas. Este investigador encontró que se les podía enseñar a aplicar al menos un método heurístico para la solución de problemas, que es mejor enseñarles heurística por sí sola que combinada con otros métodos, y que la habilidad de aplicar al menos una estrategia heurística es independiente del medio que se utiliza para dar a conocer la misma. Estos estudiantes lograron solucionar los problemas matemáticos.

Espinoza (2018) realizó una investigación cuantitativa con diseño cuasiexperimental para determinar el efecto del programa de estrategias heurísticas en la solución de problemas de matemáticas en estudiantes de segundo grado de primaria. La investigación consistió en un grupo de control y un grupo experimental, con la participación de 38 estudiantes seleccionados por muestreo por conveniencia de una población de 61 estudiantes. El grupo de control tomó la preprueba y posprueba sin recibir la aplicación del programa de estrategias heurísticas a diferencia del grupo experimental. Este investigador encontró que la aplicación del programa de estrategias heurísticas aumentó el desarrollo de las capacidades en la solución de problemas de matemáticas en estudiantes del segundo grado de primaria. A su vez, exhortó a los educadores a utilizar diferentes estrategias heurísticas y modelos en la solución de problemas.

Cabe señalar que resolver un problema es encontrar un camino allí donde no se conocía previamente camino alguno, encontrar la forma de superar una dificultad, de sortear un obstáculo, conseguir el fin deseado, que no se consigue de una forma inmediata, a partir del uso de los medios adecuados (Polya, citado por García-Jiménez, 1992). En el siglo XX, la solución de problemas estaba considerada una parte esencial de la educación en matemáticas. Mediante la solución de problemas, los estudiantes experimentan la potencia y utilidad de las matemáticas en el mundo que los rodea (García-Jiménez, 1992).

De acuerdo con Kilpatrick (1985), el éxito en la solución de problemas depende de la posesión de un extenso conocimiento sobre el dominio, las técnicas disponibles para representar y transformar el problema, y los procesos metacognitivos para monitorear y guiar el desempeño. Schroeder y Lester (1989) expresaron que el rol de la solución de problemas es hacer que el estudiante desarrolle su entendimiento de las matemáticas y debido a esto insistieron en que la enseñanza a través de la solución de problemas es el enfoque más apropiado.

La solución de problemas matemáticos es un tema de gran inseguridad para estudiantes de todos los niveles escolares (NCTM, 2000). Algunos estudiantes presentan dificultad en el proceso de solucionar problemas matemáticos incluso antes de comenzar. Para que un estudiante pueda internalizar la importancia de saber resolver un problema matemático es necesario que él mismo lo entienda en su raíz. Por medio de las estrategias heurísticas y el método de Polya se pretende instruir al estudiante en la solución de problemas de modo que entienda el problema desde antes de comenzarlo y pueda familiarizarse con él para su comprensión. Polya (1945) resaltó la importancia de entender el problema antes del proceso de asimilarlo o entender los datos que presenta el mismo. Polya propone la solución de problemas a través de cuatro pasos: entender, planificar, solucionar y verificar. La estrategia de Polya consiste en garantizar que: 1) se lea, se entienda y se analice el problema, 2) se elabore un plan o modelo para solucionarlo, 3) se ejecute el plan para solucionar el problema, y 4) se verifique la solución.

En la solución de problemas, las destrezas metacognitivas son fundamentales (Harp \& Mayer, 1998). El desarrollo de estas destrezas permite a los estudiantes codificar la naturaleza del problema en forma de representaciones mentales (modelos mentales), seleccionar los planes más adecuados para resolverlo e identificar y superar los obstáculos del proceso de solución (Davidson \& Sternberg, 1998). 


\section{PEÑA-SUREDA • COLÓN-ORTIZ • RAMOS-RULLÁN}

Aplicación de estrategias heurísticas en la solución de problemas que se modelan mediante...

El aprovechamiento se manifiesta en conocimiento, comprensiones y habilidades que el estudiante desarrolla como resultado de sus experiencias académicas (Colón, 2000). Andrade et al. (2001) definieron el aprovechamiento académico como las capacidades que reflejan lo que un individuo ha aprendido gracias a un proceso de formación. Haslett (2001) presentó evidencia de bajo aprovechamiento académico en estudiantes con promedios bajos que mostraron poca actitud positiva hacia sus maestros. NCTM (2011) presentó su postura sobre las altas expectativas de aprovechamiento de los estudiantes en matemáticas. Afirmó que tener altas expectativas hace que los estudiantes se enfoquen en tareas de conocimiento que presenten un desafío y que constantemente los lleven a desarrollar y enriquecerse de nuevos conocimientos. Esto no implica que se les adelante material o se pretenda que aceleren algún nivel académico, sino que se los impulse a pensar y a entender a cabalidad de acuerdo con el nivel académico en el que se encuentran. Es necesario fomentar la construcción de grupos de trabajo en el salón de clases para fomentar la solución de problemas y la comunicación racional; encontrarle sentido a las matemáticas es esencial para el desarrollo de altas expectativas hacia la materia (NCTM, 2011).

Pérez-López (2013) señaló que para que haya aprovechamiento académico tiene que haber un ambiente formal en el que lo aprendido constituya un objetivo de enseñanza. El aprovechamiento académico de un estudiante no se mide solamente por medio de pruebas sin considerar otros factores importantes, sin embargo, las pruebas pueden ser un mecanismo útil y viable. En el rendimiento académico influyen de manera determinante factores emocionales y afectivos.

\subsection{Preguntas de investigación}

1. ¿Cuál es el rendimiento académico en ecuaciones algebraicas de los estudiantes de un curso de matemática general de una institución educativa postsecundaria?

2. En cuanto al rendimiento académico de los conceptos de ecuaciones algebraicas, ¡cómo se comparan los grupos de estudiantes de un curso de matemática general de una institución educativa postsecundaria que están bajo estudio?

3. ¿En qué medida la aplicación de las estrategias heurísticas para la solución de problemas que se modelan mediante ecuaciones algebraicas aumentó el rendimiento académico de los estudiantes de un curso de matemática general de una institución educativa postsecundaria?

\subsection{Hipótesis}

H01: No existe diferencia significativa entre los grupos bajo investigación de rendimiento académico.

H11: Existe diferencia significativa entre los grupos bajo investigación de rendimiento académico.

H02: La aplicación de estrategias heurísticas para la solución de problemas no tiene efecto significativo en el aumento del rendimiento académico en ecuaciones en los estudiantes de un curso de matemática general de una institución educativa postsecundaria.

H12: La aplicación de estrategias heurísticas para la solución de problemas tiene efecto significativo en el aumento del rendimiento académico en ecuaciones en los estudiantes de un curso de matemática general de una institución educativa postsecundaria.

\section{Método}

\subsection{Objetivos}

1. Desarrollar unidades instruccionales en la aplicación de estrategias heurísticas para la solución de problemas que se modelan mediante ecuaciones algebraicas en un curso de matemática general de nivel postsecundario.

2. Determinar el rendimiento académico en ecuaciones algebraicas de los estudiantes de un curso de matemática general de una institución postsecundaria.

3. Comparar los grupos bajo investigación en 
términos del rendimiento académico de los conceptos medidos en la solución de problemas que se modelan mediante ecuaciones algebraicas.

4. Analizar el posible efecto del uso de estrategias heurísticas para la solución de problemas a través de unidades instruccionales en el rendimiento académico de los estudiantes de nivel postsecundario.

\subsection{Marco conceptual}

Para la solución de problemas se puede aplicar el constructivismo; el estudiante construirá la posible solución del problema matemático por medio de la utilización de la estrategia heurística y a su vez podrá compartir con sus compañeros los resultados de manera que fomente el trabajo cooperativo (Novotná et al., 2014). Dentro del enfoque constructivista se aplican estrategias de educación y a su vez al proceso de enseñanza-aprendizaje. La solución de problemas es una estrategia utilizada dentro del enfoque constructivista aplicable a cualquier materia que requiera la construcción de conocimiento (Torres, 2010). Partiendo de la premisa de que existe un problema, los estudiantes deben investigar, colaborar e intercambiar opiniones para tomar decisiones y elaborar una propuesta con el propósito de encontrar una solución (Fernández Álvarez, 2019).

El estudiante va a adquirir conocimiento sobre la solución de problemas y con la aplicación de las estrategias heurísticas solucionará los problemas matemáticos. La teoría del constructivismo es aplicable al marco conceptual de este estudio dado que el proceso de solución de problemas en la aplicación de estrategias heurísticas será guiado por el maestro, pero construido por el estudiante utilizando conceptos previos y conocimiento adquirido.

\section{Figura 1. Diagrama del marco conceptual}

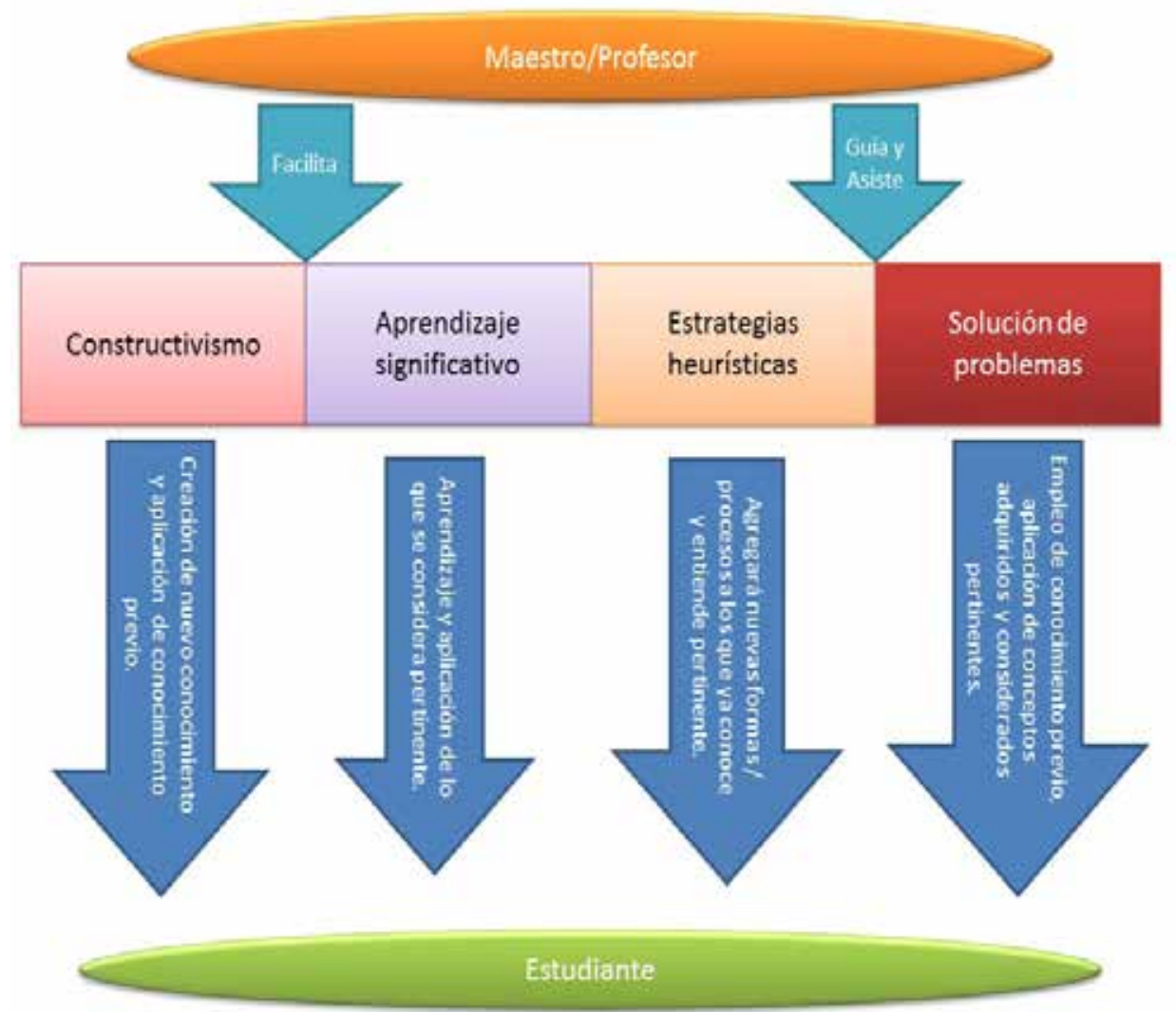

Nota: Elaboración propia. 


\section{PEÑA-SUREDA • COLÓN-ORTIZ • RAMOS-RULLÁN}

Aplicación de estrategias heurísticas en la solución de problemas que se modelan mediante...

De acuerdo con Ausubel (1986), el ser humano aprende más fácil todo lo que tiene significado para él, a partir de lo que conoce, lo que conocerá y la actitud que se tenga para ello. Para enseñar matemáticas de modo significativo, es necesario que los maestros ayuden a los estudiantes a desarrollar entendimiento de comprobación natural y la habilidad de buscar conocimiento previo para aplicarlo al nuevo.

El estudiante aprenderá todo aquello que le resulte interesante y pertinente, por tal razón es importante facilitar el proceso de la solución de problemas aplicando el uso de las estrategias heurísticas. El estudiante empleará conocimiento previo y conceptos adquiridos en el proceso, y dispondrá de una actitud positiva en el proceso de la solución de problemas que se modelan mediante ecuaciones algebraicas para tener éxito en el mismo.

\subsection{Población y muestra}

La población de investigación estuvo compuesta por estudiantes inscritos en un recinto de una institución postsecundaria del área suroeste de Puerto Rico. Los estudiantes que formaban parte de la población estaban matriculados en programas de pregrado asociados con la especialidad en campos relacionados con la salud, y en el momento de la investigación se inscribieron en el primer o segundo año académico. Los participantes estaban en el proceso de completar el curso de Matemáticas Generales, que tuvo una duración de 4 meses. Participaron en la investigación dos grupos de control y un grupo experimental con estudiantes de una institución postsecundaria. Los grupos estaban previamente conformados por los encargados de los programas académicos de la institución.

La muestra total que participó en el estudio fue de 27 estudiantes, de los cuales 11 eran el grupo de control 1, 6 eran el grupo de control 2, y 10 eran el grupo experimental. Los dos grupos de control no fueron sometidos al tratamiento de aplicación de estrategias heurísticas para la solución de problemas algebraicos, sino que recibieron clase con enseñanza tradicional alineada con el programa del curso, mientras que el grupo experimental fue sometido al tratamiento.

\subsection{Instrumento}

El instrumento consistió en una prueba de logro, la cual fue diseñada por la investigadora y validada por un comité de expertos en la materia. Se llevó a cabo un estudio piloto con el propósito de validar la prueba de logro. Esta prueba se verificó con dos análisis estadísticos: Kuder Richardson para la confiabilidad y un análisis de dificultad y discriminación. Después de estos dos análisis, la prueba se redujo de 20 problemas matemáticos a 15 . Se eliminaron 5 problemas debido a la dificultad y la discriminación. La confiabilidad de la prueba fue de 0.90 , lo que indica que fue altamente confiable según los valores propuestos por McMillan y Schumacher (2011), esto la hizo viable para su administración como preprueba para el grupo de control 1 y el grupo experimental.

\subsection{Procedimiento de recogida y análisis de datos}

Los dos grupos de control no fueron sometidos al tratamiento de estrategias heurísticas para la solución de problemas algebraicos. Ambos recibieron una clase con enseñanza tradicional alineada con el plan de estudios del curso, mientras que el grupo experimental fue sometido al tratamiento. Al grupo experimental se le enseñó a resolver ecuaciones algebraicas utilizando estrategias heurísticas con el método IDEAL de cinco pasos. De acuerdo con Phillips et al. (2017), el método IDEAL presentado por Bransford y Stein en 1987 propone alcanzar la meta de solucionar un problema a través de la utilización de estrategias y la elaboración de un plan. Bransford y Stein (1987) propusieron estrategias para la solución de problemas basadas en las propuestas por Polya en 1945. La estrategia propuesta por estos autores fuer la identificación del problema, la definición y presentación del problema, la elaboración de posibles estrategias, la actuación fundada en el procedimiento y los logros, observación y evaluación de los efectos de la actividad educativa. Dicha estrategia fue abreviada con la sigla IDEAL. Uno de los grupos de control y el grupo experimental tomaron la preprueba y la posprueba. El otro grupo de control solo realizó la posprueba. El propósito de tener dos grupos de 
control era administrar la preprueba solo a uno para evitar el efecto Hawthorne en ese grupo (McMillan y Schumacher, 2011).

El grupo de control aprendió a resolver ecuaciones de la manera tradicional, aplicando reglas comunes de solución de ecuaciones. El grupo experimental utilizó una Guía del Estudiante creada por el investigador, la cual incluía ejemplos paso a paso y procedimientos para resolver una ecuación algebraica con el método IDEAL. También se desarrolló una Guía del Profesor en la que se incluyó práctica adicional, así como material didáctico adicional para ser utilizado como guía. Después del tratamiento de cuatro semanas, los tres grupos tomaron la posprueba.

Se describirán de manera detallada las estrategias, técnicas y herramientas de investigación científica. Es decir, se describirá cómo se realizó el estudio, incluyendo las definiciones conceptuales y operacionales de las variables empleadas. La descripción completa de los métodos utilizados permitirá al lector evaluar si la metodología es apropiada, así como la confiabilidad y validez de los resultados.

\section{Resultados}

Los datos obtenidos se analizaron mediante el programa computadorizado IBM Statistical Package for the Social Sciences (SPSS), versión 26.0. Al inicio de la investigación se administró una prueba para determinar el rendimiento académico del estudiante en ecuaciones algebraicas. Los resultados de esta prueba formaron parte de todos los análisis estadísticos que se llevaron a cabo. Los grupos que tomaron esta prueba fueron el control 1 y el experimental. Las puntuaciones individuales y el cálculo de la media se consideraron para determinar el rendimiento académico en ecuaciones algebraicas de los estudiantes del curso general de matemáticas. Estos participantes fueron los que a su vez realizaron la preprueba que tuvo 15 problemas de ecuaciones. El promedio del grupo de control 1 fue del $42 \%$. El promedio del grupo experimental fue del $40 \%$. Los grupos eran comparables. Ambos grupos tenían poco conocimiento sobre la solución de problemas que se modelan mediante ecuaciones algebraicas.

Se llevaron a cabo análisis para comparar el rendimiento académico en ecuaciones algebraicas de dos de los grupos participantes en la investigación. Los grupos considerados fueron los que tomaron la preprueba: C1 y E. Para llevar a cabo esta comparación se realizó una prueba t pareada para analizar el rendimiento de los estudiantes antes y después del tratamiento en el mismo grupo; a su vez se llevó a cabo una prueba $t$ independiente para comparar el rendimiento académico de los grupos al final del tratamiento. El resultado de la prueba t pareada para el grupo de control 1 reveló que había una diferencia significativa $(\mathrm{p}<0.05)$ entre los resultados.

Al mismo tiempo, se informó de que también había una diferencia significativa en la administración de preprueba y posprueba en el grupo experimental. Aunque se encontró una diferencia significativa en los dos grupos y la del grupo experimental fue mayor.

Tabla 1. Preprueba y posprueba del grupo de control 1

\begin{tabular}{|c|c|c|c|c|c|c|c|}
\hline Variable & Prueba & $M$ & $\mathbf{N}$ & SD & $t$ & gl & $p$ \\
\hline $\begin{array}{l}\text { Aprovechamiento } \\
\text { académico en } \\
\text { matemáticas }\end{array}$ & Pre & 41.9 & 10 & 23.5 & -2.581 & 9 & .030 \\
\hline (enseñanza tradicional) & Pos & 61.9 & 10 & 23.8 & & & \\
\hline
\end{tabular}


Tabla 2. Preprueba y posprueba del experimental

\begin{tabular}{lccccccc}
\hline Variable & Prueba & M & N & SD & t & gl & p \\
\hline $\begin{array}{l}\text { Aprovechamiento } \\
\begin{array}{l}\text { académico en } \\
\text { matemáticas }\end{array}\end{array}$ & Pre & 40.1 & 10 & 25.1 & -5.246 & 9 & .001 \\
\cline { 2 - 7 } $\begin{array}{l}\text { (estrategias } \\
\text { heurísticas) }\end{array}$ & Pos & 79.3 & 10 & 13.4 & & & \\
\hline
\end{tabular}

Tabla 3. Prueba ANOVA para la posprueba de los tres grupos: C1, C2 y E

\begin{tabular}{|c|c|c|c|c|c|}
\hline ANOVA & & & & & \\
\hline Fuente & $\begin{array}{c}\text { Suma de } \\
\text { cuadrados }\end{array}$ & gl & $\begin{array}{l}\text { Cuadrado } \\
\text { Medio }\end{array}$ & $F$ & $p$ \\
\hline Entre grupos & 2862.005 & 2 & 1431.003 & 3.573 & 0.045 \\
\hline Dentro de grupos & 9211.533 & 23 & 400.501 & & \\
\hline Total & 12073.538 & 25 & & & \\
\hline
\end{tabular}

$\mathrm{Al}$ administrar un preprueba al grupo de control 1 y al grupo experimental se pretendía hacer una comparación de los resultados para determinar el rendimiento académico de cada uno de los grupos antes de ser sometidos al tratamiento. Los resultados mostraron que los dos grupos tenían similar rendimiento académico con respecto a las ecuaciones algebraicas. El grupo de control 1 y el grupo experimental eran comparables en cuanto a los resultados de la preprueba.

La comparación de los promedios posteriores a la prueba para ambos grupos también reveló que la diferencia era superior al 19\%. El grupo de control 1 tenía una media de 61 en la administración posterior a la prueba, mientras que el grupo experimental tenía alrededor de 80. Si hay una diferencia significativa de acuerdo con los resultados de la prueba t pareada esto no implica que el rendimiento académico de los estudiantes participantes sea bueno ni que se exhibiera un mayor rendimiento, pero todavía se puede clasificar como deficiente dado que el porcentaje mínimo requerido para aprobar el curso es del 65\%.

Después de que se llevó a cabo el tratamiento, se administró la posprueba para determinar cómo la aplicación de estrategias heurísticas aumentó el rendimiento de los estudiantes. Se analizaron los resultados de la posprueba de los tres grupos participantes utilizando la prueba estadística para la comparación de varianzas, ANOVA. Los resultados de la prueba mostraron una diferencia significativa, por lo que fue rechazada la hipótesis nula. Los resultados de la posprueba administrada a los grupos participantes (control 1, control 2 y experimental) revelaron que había una diferencia significativa en el rendimiento académico de los estudiantes en la resolución de problemas que se modelan mediante ecuaciones algebraicas después de ser tratados. Aunque hubo una diferencia tanto en el grupo de control 1 como en el grupo experimental después del tratamiento, el rendimiento académico de los estudiantes del grupo experimental fue mayor.

Para determinar el posible efecto del uso de las estrategias heurísticas, al considerar los resultados de la posprueba de los tres grupos bajo investigación, se realizó una prueba ANOVA. Los resultados de dicha prueba están expresados en la Tabla 3.

Se realizó un análisis de comparaciones múltiples a través de una t-independiente de los resultados de 
la posprueba de los tres grupos en estudio. A través de este análisis, se determinó el rechazo o no rechazo de la hipótesis nula para determinar si había una diferencia entre los grupos en cuanto a rendimiento académico. En la comparación de los resultados de la posprueba del grupo de control 1 y el grupo de control 2 no se encontró ninguna diferencia significativa $(p>0,05)$, sin embargo, en la comparación del grupo experimental con los grupos de control 1 y 2 hubo una diferencia significativa $(p<0.05)$. Esto implica que el grupo experimental —que recibió el tratamiento de la aplicación de estrategias heurísticas para resolver problemas de ecuaciones algebraicas - demostró una mayor ventaja académica que los grupos de control —que recibieron la enseñanza tradicional- El uso de estrategias heurísticas utilizando el modelo IDEAL aumentó el rendimiento académico de los estudiantes que participaron en el tratamiento. Para que el estudiante pueda asimilar el problema y tratar de resolverlo es necesario que pueda entenderlo (Polya, 1945). El método IDEAL se aplica para identificar, presentar y entender el problema antes de intentar emplear algún proceso para resolverlo. Al comparar el rendimiento de los estudiantes de los grupos de control, se puede determinar que el uso de las estrategias fue eficaz para aumentar el rendimiento académico de los estudiantes después del tratamiento.

En la Tabla 4 se muestran los resultados de la prueba $\mathrm{t}$-independiente para los resultados de la preprueba de los grupos $\mathrm{C} 1$ y E.

En la Tabla 5 se incluyen los resultados de las múltiples comparaciones entre los resultados de la administración de la posprueba para los tres grupos participantes en la investigación.

Tabla 4. Resultados de la preprueba de los grupos C1 y E

\begin{tabular}{cccccccc}
\hline Variable & Grupo & M & N & SD & T & gl & p \\
\hline Aprovechamiento académico & C1 & 40.1 & 10 & 25.1 & -0.270 & & 19 \\
$\begin{array}{c}\text { en matemáticas (estrategias } \\
\text { heurísticas) }\end{array}$ & E & 42.9 & 11 & 22.5 & & & \\
\hline
\end{tabular}

Tabla 5 Múltiples comparaciones de la posprueba entre los tres grupos: C1, C2 y E

\begin{tabular}{|c|c|c|c|c|}
\hline \multicolumn{5}{|c|}{ Múltiples comparaciones } \\
\hline \multirow{2}{*}{\multicolumn{2}{|c|}{ Grupos }} & \multirow[b]{2}{*}{$\mathbf{P}$} & \multicolumn{2}{|c|}{ Intervalo al $95 \%$ de confiabilidad } \\
\hline & & & Cota inferior & Cota superior \\
\hline \multirow{2}{*}{$\mathrm{C} 1$} & $\mathrm{C} 2$ & 0.591 & -15.75 & 27.01 \\
\hline & $E$ & 0.045 & -37.51 & -0.49 \\
\hline \multirow{2}{*}{$\mathrm{C} 2$} & $\mathrm{C} 1$ & 0.591 & -27.01 & 15.75 \\
\hline & $E$ & 0.026 & -46.01 & -3.25 \\
\hline \multirow{2}{*}{$\mathrm{E}$} & $\mathrm{C} 1$ & 0.045 & 0.49 & 37.51 \\
\hline & $\mathrm{C} 2$ & 0.026 & 3.25 & 46.01 \\
\hline
\end{tabular}


De acuerdo con los resultados presentados en la Tabla 5, existió diferencia entre algunos de estos tres grupos.

\section{Discusión y conclusiones}

Los estudiantes de nivel post secundario, como los de esta investigación, deben poseer al menos un conocimiento básico sobre cómo solucionar ecuaciones algebraicas de primer grado; sin embargo, la materia de Matemáticas les puede causar apatía y la solución de problemas ser una de esas competencias en las que tienen mayor dificultad (Meneses y Peñaloza, 2019).

Los resultados de la administración de la prueba de aprovechamiento indican que estos estudiantes no dominan las destrezas de solución de problemas que se modelan mediante ecuaciones algebraicas. Por ende, los estudiantes no demostraron tener las herramientas o conocimiento para solucionar los problemas de ecuaciones algebraicas, similar a lo que expuso Hernández (2002) en su investigación. Este autor realizó una investigación con estudiantes de los primeros dos ańos de universidad de una institución universitaria privada de Puerto Rico. Hernández observó dos elementos que impedían a los estudiantes construir representaciones matemáticas en la solución de problemas. Los estudiantes que participaron en la investigación de Hernández tuvieron dificultad para establecer la relación entre los datos que le provee el problema y la capacidad de identificar los conceptos que deben aplicar para solucionarlo. Esto impedía que el estudiante creara una estrategia para solucionar el problema, impulsándole a utilizar una estrategia general proveniente de su conocimiento previo. Cuando se dificultaba el proceso de solución para estos estudiantes, descartaban la opción de utilizar una nueva estrategia de solución. Similar a los resultados de los estudiantes de esta investigación, luego de tomar la preprueba en el que ambos grupos demostraron no dominar el concepto de las ecuaciones algebraicas lo que implica que utilizaban conocimiento previo para solucionar las ecuaciones algebraicas. En la corrección de las pruebas para el análisis estadístico se encontró que los estudiantes no conocían procesos claros de solución que fundamentaran la respuesta dada, lo que demostraba que no tenían dominio de los procesos de solución. Se propuso la aplicación de estrategias para solucionar ecuaciones, lo que coincide con los hallazgos de Hernández (2002), el cual sugirió el uso de un modelo para enseñar a solucionar ecuaciones. En ambas investigaciones se pretendió facilitar y enseñar a los estudiantes a solucionar problemas matemáticos al utilizar métodos nuevos.

La solución de problemas debe trascender más allá de los algoritmos y es necesario que en el salón de clases se fomente el uso de estrategias (García-García, 2019). Los docentes deben impulsar a los alumnos a considerar métodos o modelos para la solución de problemas señalando que se debe trabajar en etapas, fases o pasos que se puedan modificar y ajustar a las necesidades del proceso de solución (Chandia et al., 2016). El apoyo del docente en el proceso de solución de problemas es fundamental para mejorar el rendimiento académico de la materia y el dominio y comprensión del tema.

Los hallazgos de la investigación realizada por Annable (2006) concuerdan con la diferencia de rendimiento académico del grupo de control 1 y el grupo experimental. En su investigación, Annable enseñó matemática a estudiantes de sexto grado basado en solución de problemas. En los resultados se evidenció un aumento del desarrollo del pensamiento crítico de los estudiantes y aumentó su desempeño en la solución de problemas. De acuerdo con este investigador, en la solución de problemas se recalcó la utilización de estrategias para la solución de problemas y cuando los estudiantes discutieron los problemas con sus compañeros, las destrezas en la solución de problemas aumentaron. Similar a los resultados de esta investigación, en la que se evidenció que los estudiantes aumentaron el rendimiento académico en la solución de problemas que se modelan mediante ecuaciones algebraicas cuando aplicaron las estrategias heurísticas. El uso de estrategias heurísticas para solucionar problemas favorece el rendimiento académico de los estudiantes y fomenta el pensamiento creativo (Espinoza, 2018; Mendoza, 2018). 
La utilización de procesos heurísticos permite que los estudiantes con habilidades matemáticas y aquellos con resistencia hacia la asignatura participen en el proceso de proponer alternativas de solución debido a que no se limita a un procedimiento especifico (Prado-Durán, 2017). El uso de estrategias para la solución de problemas debe ser considerado un tema de libre acceso a todos los estudiantes sin importar sus habilidades matemáticas. El proveer herramientas para facilitar el proceso de solución de problemas a estudiantes poco motivados en la materia contribuye a mejorar su rendimiento académico. Es posible considerar la solución de problemas como uno de los ejes principales del aprendizaje de la matemática y de la formación académica en general (Arias et al., 2018).

Es importante que se haga uso de las etapas o procesos heurísticos para la solución de problemas porque fomentan la ideación y la búsqueda de vías para llegar a una solución (Barrantes et al., 2016). El método de Polya le permite al estudiante reflexionar sobre el problema que se pretende resolver, ya que en cada etapa del proceso de solución se analiza si el método es viable. Cuando el estudiante trabaja para solucionar un problema, él mismo muestra interés por implementar su conocimiento en el área y refleja la aplicabilidad de los conocimientos adquiridos, entre ellos, los proceso para la solución de problemas (Cárdenas \& González, 2016).

De acuerdo con Stinson y Claus (2000), la forma tradicional de enseñanza es un proceso que se utiliza para educar a los estudiantes de forma regular o tradicional en el que es posible la utilización de métodos de enseñanza que fomentan un proceso de enseñanza-aprendizaje de calidad. De acuerdo con el planteamiento de estos autores, la enseńanza a partir del uso de una forma tradicional es posible, lo indispensable es que sea de calidad. Los estudiantes del grupo de control 1 tuvieron mejor desempeńo, es evidente que hubo un proceso de aprendizaje efectivo. La enseñanza tradicional se centra en el aprendizaje de hechos y reglas establecidas rigurosamente, sin embargo, utilizar nuevos métodos es necesario para aprender nuevos conocimientos (Pehkonen et al., 2013). En ocasiones, la enseñanza tradicional puede resultar un proceso sistemático y rígido. Por tal razón, Stinson y Claus (2000) afirmaron que los métodos o las estrategias de enseńanza que se utilizan no son adecuados para todos los estudiantes. Por lo tanto, cabe señalar que en la presente investigación el grupo de control 1 mejoró su desempeño en la solución de problemas que se modelan mediante ecuaciones algebraicas, sin embargo, no presentó resultados significativos como los del grupo experimental. Los estudiantes del grupo de control 1 aprendieron a solucionar ecuaciones algebraicas con el método de enseńanza tradicional, lo cual aportó al mejoramiento y enriquecimiento de conocimiento, sin embargo, el uso de estrategias heurísticas favoreció significativamente al grupo experimental. No se debe desestimar la enseñanza tradicional y es necesario resaltar su importancia en los procesos de aprendizaje, sin embargo, la enseñanza podría diseñarse considerando la compresión inicial que tengan los estudiantes sobre el tema y establecer conexiones entre el conocimiento previo y los nuevos procesos (Caviedes et al., 2019).

Presentar el tema de la solución de problemas en los salones de clase es una necesidad para muchos sistemas educativos del mundo, al coincidir en que es parte fundamental del currículo de matemáticas (Felmer \& Perdomo-Diaz, 2017). Los maestros reconocen la necesidad de dar prioridad a la solución de problemas y de enfatizar en sus aplicaciones en la vida diaria. La solución de problemas puede darse de forma creativa en la que el maestro, como facilitador, fomente la elaboración de un plan de solución. El plan para solucionar el problema puede ser inefectivo al aplicarse por primera vez, por lo tanto, queda del estudiante trabajar en colaboración con el conocimiento previo para modificar el plan y aplicar estrategias. Cabe señalar que el uso del método IDEAL para la solución de problemas que se modelan mediante ecuaciones algebraicas es una de muchas herramientas que fomentan el pensamiento crítico, la elaboración de un plan y el trabajo colaborativo para la solución de problemas. Las etapas del método dieron oportunidad a los estudiantes del grupo experimental de la presente investigación a identificar el problema, desarrollar estrategias, elaborar el plan, aplicarlo y verificar los resultados. 
El uso de las estrategias heurísticas a través del uso del modelo IDEAL aumentó el rendimiento académico de los estudiantes participantes del tratamiento. El método IDEAL implica identificar, presentar y entender el problema antes de tratar de emplear algún proceso para solucionarlo. Al comparar el desempeño de los estudiantes de los grupos de control se puede determinar que el uso de las estrategias fue efectivo al aumentar el rendimiento académico de los estudiantes después del tratamiento. Esto es parte de los primeros dos pasos del método IDEAL, lo que apoya el uso de este método no tradicional en esta investigación. EL modelo IDEAL no es perfecto, sin embargo, ayuda a identificar y reconocer lo que se debe considerar para resolver el problema (Zona-Lopez \& Giraldo-Márquez, 2017).

Después de discutir los resultados de esta investigación, llegamos a la siguiente conclusión: el rendimiento académico de los estudiantes en la solución de problemas que se modelan mediante ecuaciones algebraicas aumentó significativamente con el uso de estrategias heurísticas, tanto los métodos de enseñanza tradicionales como los no tradicionales ayudaron a los estudiantes a alcanzar un mejor rendimiento en el curso, sin embargo, el rendimiento de los estudiantes en el grupo experimental fue notable.

El análisis de datos de la administración de la preprueba reveló que los participantes tenían conocimientos similares sobre la algebraica, el análisis de los datos de la gestión de la posprueba del grupo de control 1 y del grupo experimental mostró que en ambos grupos había una diferencia significativa después del tratamiento y los resultados de la administración de la posprueba de los tres grupos revelaron que había una diferencia significativa entre los tres grupos en la solución de ecuaciones algebraicas, por lo que se puede sugerir que el tratamiento fue efectivo.

Las matemáticas son un mundo amplio que goza de la oportunidad de su aplicación a la vida diaria. En muchas ocasiones, los estudiantes no identifican la conexión de las matemáticas con la vida diaria y cómo se puede aplicar en el futuro. Parte de la labor del maestro es alimentar el pensamiento creativo del estudiante proveyendo herramientas accesibles para impulsar el éxito en la materia. Cuando el maestro le facilita al estudiante las herramientas que puede utilizar para crear procesos y posibles soluciones, pone el mundo a sus pies. Los maestros deben estar en continuos procesos de capacitación y actualización para que sus prácticas pedagógicas se vean continuamente enriquecidas (Meneses \& Peñaloza, 2019). Cárdenas et al. (2016) reportaron que los docentes centran mayormente sus evaluaciones en procesos de solución mecánicos. Los hallazgos de esta investigación exhortan a los maestros a educarse en la aplicación de estrategias para la solución de problemas para así facilitar herramientas a los estudiantes e impulsar altos niveles de pensamiento crítico. El uso de estrategias heurísticas para la solución de problemas es una herramienta que fomenta descubrir, elaborar y analizar un plan e impulsa al estudiante a tener éxito en la solución de un problema matemático.

\section{Referencias bibliográficas}

Andrade M., Freixas, I., \& Miranda, C. (2001). Predicción del rendimiento académico lingüístico y lógico matemático por medio de las variables modificables de las inteligencias múltiples del hogar. Boletín Investigación Educacional, 16, 301-315.

Annable, C. J. (2006). Developing critical thinking skills and mathematical problem-solving ability in grade six students. [Tesis de maestría, Nippising University, Canadá]. ProQuest Dissertations and Theses UMI No. MR27029.

Arias-Rueda, J. H., Arias, M., \& Urdaneta, M. (2018). Estrategias heurísticas en resolución de problemas a través de una experiencia integradora. Revista Sarance, 42, 43-64.

Ausubel, D. P. (1986). Sociología Educativa: un punto de vista cognoscitivo. México: Editorial Trillas.

Barrantes-Fajardo, L., Cruz-Contreras, M. R., \& Gutiérrez-Montaña, R. E. (2016). La heurística como estrategia de enseñanza creativa en la resolución de problemas matemáticos relacionados con el pensamiento numérico de los estudiantes del ciclo tres grados sextos 
del Colegio Arborizadora Baja IED. [Tesis de maestría, Universidad de la Salle, Bogotá].

https://r.issu.edu.do/l? $1=106082 \mathrm{yt}$

Bransford, J. D., \& Stein, B. S. (1987). Solución I.D.E.A.L. de problemas. Guia para mejor pensar, aprender y crear. Barcelona: Labor.

Benson-O'Connor, C., McDaniel, C., \& Car, J. (2019). Bringing Math to Life: Provide Students Opportunities to Connect their Lives to Math, $\mathrm{Ne}$ tworks: An Online Journal for Teacher Research, 21(2). https://r.issu.edu.do/l?l=10607LPH

Calucag, L. (2016). Divergence of Scientific Heuristic Method and Direct Algebraic Instruction. Journal of Education and Practice, 7(3), 131-135.

https://eric.ed.gov/?id=EJ1089794

Cárdenas, C., \& González, D. (2016). Estrategia para la resolución de problemas matemáticos desde los postulados de Polya mediada por las TIC, en estudiantes del grado octavo del Instituto Francisco José de Caldas. [Tesis de maestría, Universidad Libre de Colombia]. https://r.issu.edu.do/l?l=10606cy6

Cárdenas-Lizarazo, J., Blanco-Nieto, L., Guerrero-Blanco, E., \& Caballero-Carrasco, A. (2016). Manifestaciones de los Profesores de Matemáticas sobre sus Prácticas de Evaluación de la Resolución de Problemas. Bolema, Rio Claro (SP), 30(55), 649-669. http://dx.doi.org/00.1590/1980-4415v30n55a17

Caviedes-Barrera, S., De Gamboa-Rojas, G., \& Badillo-Jiménez, E. (2019). Conexiones matemáticas que establecen maestros de formación al resolver tareas de medida y comparación de áreas, Praxis, 15(1), 69-87. http://dx.doi.org/10.21676/23897856.2984

Chandia, E., Rojas, D., Rojas, F., \& Howard, S. (2016). Creencias de formadores de profesores de matemática sobre la resolución de problemas. Bolema, Rio Claro (SP), 30(55), 605-624.

http://dx.doi.org/00.1590/1980-4415v30n55a15

Common Core State Standards Initiative. (2018). Standards for Mathematical Practices. https://r.issu.edu.do/l?l=10605AiZ
Davidson, J. E., \& Sternberg, R. J. (1998). Smart problem solving: How metacognition helps. Metacognition in educational theory and practice. Mahwah, NJ: Erlbaum.

Espinoza Aguilar, J. A. (2018). El programa estrategias heuristicas en la resolución de problemas matemáticos en estudiantes del segundo grado de primaria de la I.E. 1025 El Agustino 2016. [Tesis de posgrado, Universidad Cesar Vallejo, Perú].

https://r.issu.edu.do/l?l=10604gH4

Espinoza González, J. (2017). La resolución y planteamiento de problemas como estrategia metodológica en clase de matemática. Atenas, 3(39), 64-72. https://r.issu.edu.do/l?l=10603XRe

Felmer, P., \& Perdomo-Díaz, J. (2017). Un programa de desarrollo profesional docente para un currículo de matemática centrado en las habilidades: la resolución de problemas como eje articular. Educación Matemática, 29(1), 201-217.

https://doi.org/10.24844/em2901.08

Fernández-Álvarez, R. (2019). La enseñanza del paisaje desde una concepción constructivista: propuesta didáctica. DEDICA. Revista de Educación e Humanidades, 15, 135-159.

https://r.issu.edu.do/l?l=10602MrM

García-García, J. (2019). Estrategias en la resolución de problemas algebraicos en un contexto intercultural en el nivel superior. Bolema, Rio Claro (SP), 33(63), 205-225.

http://dx.doi.org/10.1590/1980-4415v33n63a10

García-Jiménez, J. E. (1992). Ideas, pautas y estrategias heurísticas para la solución de problemas. Revista Aula de Innovación Educativa, 6(01), 14-21.

Gurat, M. (2018). Mathematical problem-solving strategies among student teachers, Journal on Efficiency and Responsibility in Education and Science, 11(3), 53-64. https://doi.org/10.7160/eriesj.2018.110302.

Harp, S., \& Mayer, R. (1998). How Seductive Details Do Their Damage: A Theory of Cognitive Interest in Science Learning. Journal of Educational Psychology, 90(3), 414-434. https://r.issu.edu.do/l?l=10914813. 
Haslett, B. (2001). Influence on student ability and sex on student's attitude toward teachers. University of Delaware. USA: Department of Speech Communication.

Hernández-Rodríguez, O. (2002). Procesos cognoscitivos y metacognoscitivos en estudiantes universitarios puertorriqueños en la solución de problemas matemáticos no típicos [Tesis doctoral, Universidad de Puerto Rico]. ProQuest Dissertations and Theses UMI No. 305495844 .

Kilpatrick, J. (1985). A retrospective account of the past 25 years of research on teaching mathematical problem solving. Hillsdale, NJ: Erlbaum.

Lesh, R., \& Zawojewski, J. S. (2007). Problem solving and modeling. The Second Handbook of Research on Mathematics Teaching and Learning. Charlotte, N.C: Information: F.K. Lester Jr.

McMillan, J. H., \& Schumacher, S. (2011). Research in Education: Evidence-Based Inquiry (7th edition). Pearson: Addison Wesley.

Mendoza, L. (2018). Estrategias heurísticas para incrementar la capacidad de resolución de problemas en estudiantes de educación secundaria. Sciéndo, ciencia para el desarrollo, 21(2), 205-211. https://doi. org/10.17268/sciendo.2018.021.

Meneses, M., \& Peńaloza, D. (2019). Método de Pólya como estrategia pedagógica para fortalecer la competencia resolución de problemas matemáticos con operaciones básicas. Zona Próxima, 31, 7-25. https://doi.org/10.14482/zp.31.372.7.

National Council of Teachers of Mathematics Commission on Standards for School Mathematics. (1989). Curriculum and Evaluation Standards for School Mathematics. Reston, VA.

National Council of Teachers of Mathematics. (2019). Principles, Standards, and Expectations. https://www.nctm.org/Standards-and-Positions/Principles-and-Standards/ Principles,-Standards,-and-Expectations/

National Commission on Mathematics and Science
Teaching for the $21^{\text {st }}$ Century. (2000). Before it's too late. U.S. Department of Education. Washington, DC.

National Council of Teachers of Mathematics. (2011). High Expectations: A Position of the National Council of Teachers of Mathematics.

https://r.issu.edu.do/l?l=10601suW

Novotná, J., Eisenmann, P., Pribyl, J., Ondrusova, J., \& Brehovsky. (2014). Problem solving in school mathematics based on heuristic strategies. ERIES Journal, 7(1), 1-6.

https://doi.org/10.7160/eriesj.2014.070101.

Oladunni, M. O. (1998). An experimental study on the effectiveness of metacognitive and heuristic problem solving techniques on computational performance of students in mathematics. International Journal of Mathematical Education in Science and Technology. 29(6), 867-874.

https://doi.org/10.1080/0020739980290608

Pehkonen, E., Näveri, L., \& Laine, A. (2013). On Teaching Problem Solving in School Mathematics. CEPS Journal, 3(4), 9-23. https://r.issu.edu. do/l?l=10919ai9.

Pereira-Mendoza, L. (1980). The Effect of Teaching Heuristics on the Ability of Grade Ten Students to Solve Novel Mathematical Problems. The Journal of Educational Research, 73(3), 139-144.

https://doi.org/10.1080/00220671.1980.10885223

Pérez-López, E. I. (2013). Efecto del aprendizaje cooperativo en el aprovechamiento académico en ciencias en las destrezas de negociación de estudiantes de noveno grado de una escuela pública de la región sur de Puerto Rico. [Tesis de doctorado, Pontifica Universidad Católica de Puerto Rico].

Phillips, J., Clemmer, K., McCallum, J., \& Zachariah, T. (2017). A problem-solving framework to assist students and teachers in STEM courses. Journal of College Science Teaching, 46(4), 33-39. https://www.jstor.org/stable/44579911

Polya, G. (1945). How to solve it: a new aspect of mathematical method. Princeton, New Jersey: Princeton University Press. 
Prado-Durán, W. (2017). Heurísticos: una herramienta de razonamiento en la enseńanza y el aprendizaje de la Matemática. Conocimiento Educativo, 5, 29-40. https://doi.org/10.5377/ce.v5i0.8072.

Rodríguez, M., Gregori, P., Riveros, A., \& Aceituno, D. (2017). Análisis de las estrategias de resolución de problemas en matemática utilizadas por estudiantes talentosos de 12 a 14 años. Educación Matemática, 29(2), 159-186. https://r.issu.edu.do/l?l=10600wyH

Romberg, T. A. (1998). Comments: NCTM's curriculum and evaluation standards. Teachers College Record, 100(1), 8-21.

Santaló, L. (1995). Matemática para no matemáticos. En C. Parra \& I. Saiz (Eds.), Didáctica de matemáticas. Aportes y Reflexiones (pp. 21-38). Buenos Aires: Paidos.
Stinson, B. M., \& Claus, K. (2000). The effects of electronic classrooms on learning English composition: a middle ground between traditional instruction and computer-based instruction. The Journal, 27(7), 98-102. https://r.issu.edu.do/l?l=10920Tan.

Torres-Bonilla, L. (2010). Constructivismo pedagógico: estrategias de enseñanza de maestras y maestros del sistema público de Puerto Rico. [Tesis doctoral, Universidad de Puerto Rico]. ProQuest Dissertations and Theses. UMI No. 854342445.

Zona-López, J. R., \& Giraldo-Márquez, J. D. (2017). Resolución de problemas: escenario del pensamiento crítico en la didáctica de las ciencias. Revista Latinoamericana de Estudios Educativos, 13(2), 122-150. https://r.issu.edu.do/l?l=10921rfU.

\section{CÓMO CITAR:}

Peña-Sureda, A., Colón-Ortiz, A., \& Ramos-Rullán, I. (2021) Aplicación de estrategias heurísticas en la solución de problemas que se modelan mediante ecuaciones algebraicas en estudiantes de una institución educativa. RECIE. Revista Caribeña de Investigación Educativa, 5(2), 144-158.

https://doi.org/10.32541/recie.2021.v5i2.pp144-158 